\title{
Awareness and response to Coronavirus disease (COVID-19) epidemic among the Arab population in the Eastern Mediterranean Region and Arab Peninsula
}

\author{
Mhammad Saleh', Marwan O M Saleh², Mohammed Nabil Zahid
}

\author{
${ }^{1}$ Department of Prosthodontics, Faculty of Dentistry, Near East University, Nicosia, Turkish Republic of Northern Cyprus \\ ${ }^{2}$ Faculty of Medicine, Near East University, Nicosia, Turkish Republic of Northern Cyprus \\ ${ }^{3}$ Department of Preventive Dentistry, Prince Sattam Bin Abdalaziz University, Kingdom Saudi Arabia. \\ Corresponding author: Mhammad Saleh (E-mail: dr.m.salehh@gmail.com)
}

\begin{abstract}
Objectives: The Coronavirus (COVID-19) causes fatal symptoms especially in areas with poor medical care. This online survey planned to analyze the knowledge and apprehension about coronavirus among the Arab populations.

Methods: A cross-sectional questionnaire-based survey was conducted from 15 May 2020 to 27 May 2020. The survey included a total of 443 Arab participants. Divided into four groups according to the age; under 20 years old (28 participants), $20-40$ (359), 40-60 (49), and 7 participants were over 60 . According to gender; male (318 participants) and female (125). Based on education level, participants were categorized as a secondary school (5 participants), high school (28), university graduated (327), and postgraduate (83).

Results: Most of the participants showed a good adaptation for the precautions concerning isolation and quarantine. 299 participants stayed at home during the COVID-19 outbreak by taking a break from jobs or performing their jobs from home. 144 participants performed partial or full-time jobs from 20 to 60 groups of age. Most of the population were dependent on social media to receive the update about the virus. 141 participants said that they are not up-to-date enough about the COVID-19 related to the language barrier.

Conclusion: The majority of the participants had heard about COVID-19 and were aware of the infection control measures. Most of the participants strictly adapted to quarantine during the outbreak. Further steps need to be taken to enhance the social media accounts and internet websites in the Arabic language which concern medical and educational content.

Keywords: COVID-19, EMR, respiratory infection, aerosol infection.
\end{abstract}

\section{Introduction}

The COVID-19 has considered an international emergency problem threatening public health on the 30 of January $2020 .^{1}$ Coronaviruses are important human and animal pathogens which can cause diseases such as the Middle East Respiratory Syndrome (MERS) and the Severe Acute Respiratory Syndrome (SARS). ${ }^{2,3}$ A novel coronavirus was identified as the cause of a cluster of pneumonia cases in Wuhan, a city in the Hubei Province of China. ${ }^{1}$ In December 2019, 27 cases of pneumonia were recorded in that City. Wuhan is a very crowded city in China, the first recorded 27 patients were having clinical symptoms like dry cough, dyspnea, fever, and bilateral lung infiltrates on imaging. ${ }^{4}$ The center of this infection problem is linked to Wuhan's Huanan Seafood Wholesale Market, dealing with fish and many other animals including poultry, bats, marmots, and snakes. The local medical center makes the required investigation for the patient and approves that the 27 patients have a Severe Acute Respiratory Syndrome Coronavirus 2 (SARS-CoV-2). ${ }^{4}$ The disease was named COVID-19 by the World Health Organization (WHO). ${ }^{5}$ Despite that, most of the infected cases have spontaneous recovery but the other developed fatal symptoms include organ failure, septic shock, pulmonary edema, severe pneumonia, and Acute Respiratory Distress Syndrom (ARDS). ${ }^{6}$ Most of the cases which were suffering from fetal symptoms were from the old age group and the people who have other medical issues including cardiovascular, cerebrovascular, endocrine, digestive, and respiratory disease. ${ }^{7}$ Then, it rapidly spread over the adjacent cities, resulting in an epidemic throughout China, followed by an increasing number of cases in other countries around the world. The WHO declared the Chinese outbreak of COVID19 to be a Public Health Emergency of International Concern posing a high risk to countries with vulnerable health systems.

The emergency committee in the WHO announced that following strict instructions related to isolation, provide the social distance, quarantine for the children, elder peoples, and for those who have symptoms of pneumonia and fever have a role to prevent the spread of COVID-19 infection. ${ }^{6}$ According to the WHO report, ${ }^{8} \mathrm{a} € 10,000,000$ research fund to contribute to more efficient clinical management of patients infected with the virus, as well as public health preparedness and response. The United Kingdom government offers $£ 20,000,000$ to develop a vaccine for COVID-19.8,

Despite the preventive recommendations to prevent the transition of virus infection in the countries, the governments in most countries around the world have suspended all entry of immigrants and non-immigrants having traveled to high-risk zones. ${ }^{10}$ Hong Kong has also suspended several public transport services across the border and many hospital workers and civil servants are currently on strike. Strikers are demanding that the border to mainland China be closed completely to prevent further COVID-19 transmission. ${ }^{11}$ According to the last announcement for the WHO, the General Director of the organization announced that until the 9 June, 7 million cases have been reported of COVID-19 and 4000 deaths. Until the 9 June, new COVID-19 has been recorded from different countries including America and South Asia. Most countries 
in Africa express increasing cases, Eastern Europe and Central Asia record some increase in the last days. Until the day of writing this paper (11 June 2020) Related to the report of the world health organization in the Eastern Mediterranean region the total report cases were 687,464 and death cases were 15,405 and the daily notification of confirmed COVID-19 in EMR was stably increasing until the day of writing this articles.

The route of the transition of this infection depends on inhaling the airborne droplets or touching surfaces contaminated with the virus and then touching the mucosal membrane in the mouth, nose, or eye. For this reason, the preventive methods depending on isolation and avoiding the crowding area and following the general hygiene instruction are very important to be followed. ${ }^{12}$ These instructions were very simple to be applied but they need cooperation and a full understanding of people to stop spreading this infection.

The risk of contamination will be more in areas where people speak different languages, the language barrier will prevent the ability for these people to be up-to-date with the everyday news and regulations which are important to be followed. With all the restrictions, which have been applied by the governments to apply home isolation to prevent viral infection spread of COVID-19, the people will stay in need to get out for their needs.

All hospital and dental clinics continue to accept emergency cases during the break, and in these situations, the cooperation of the people and following the preventive instruction is very important to prevent the transition of infection. The dental clinics considered as the most high-risk area to spread infection of COVID-19, according to the WHO; ${ }^{6}$ moreover, the dental clinic could be a riskier environment for spreading the virus because of the close contact with patients and the nature of the dental treatment. ${ }^{7}$ Although patients diagnosed with COVID-19 are not supposed to receive dental treatments, dental emergencies can occur, and close contact would be unavoidable. Furthermore, both the relatively prolonged incubation period of the disease (the median incubation period was estimated to be 14 days before any symptoms could even be detected), which could increase the transmission of the disease during these lay periods. ${ }^{13}$

In this study, a survey has released to evaluate the level of knowledge of the Arab people who are living in different countries and to evaluate the response of these people when they are being in a high-risk area like dental clinics as an example, to be able to evaluate the adaptability of these people with preventive instructions of the governments and health organizations.

\section{Material and Method}

A cross-sectional questionnaire-based survey was conducted from 15 May 2020 to 27 May 2020. Ethical clearance was obtained from the institutional review board of Prince Sattam Bin Abdulaziz University (PSAU2020018). The online questionnaire planned to be shared over the social media platforms (Facebook, Instagram, and Whatsapp) to reach the maximum number of participants who belong to Arabic ethnic origin and speak Arabic as a mother language. The questions in the survey written in the Arabic language to be sure that all the participants are from the Arab population. The questionnaire consisted of 16 self-prepared questions and had 4 parts. The first part gathered personal information (age, gender, education, and career). The second part is designed to evaluate the ability of the population to understand the new regulations related to COVID-19 and see the level of the response to these regulations. In the third part, we ask the population questions about the sources they depend on to be up to date to the regulations related to COVID-19 and to evaluate the type of sources which the Arabic native speaker is able to follow. According to the report of the World Health Organization, the dental clinic and dentistry as a career classified as one of the highest risks to spread the COVID-19. For this reason, we used dental clinics as an example in the fourth part of the survey. This part of the questionnaire designed to evaluate the response of the population and evaluate their knowledge about the new regulations related to visiting dental clinics during the outbreak.

Descriptive static analysis performed for participants' characteristics (gender, age, and level of education). The univariate linear model analysis performed to see the effect of age and level of education as an independent variable on the awareness about the COCID-19 as an outcome (dependent variable). Statistical analysis was performed using version 23 of SPSS Statistics (IBM SPSS Statistics, New York, United States).

\section{Result}

In the present study, we included a total of 443 Arab participants living in different countries of EMR. According to age, participants were categorized in 4 groups as under 20 years old (28 participants), between 20 and 40 years old (359 participants), between 40 and 60 years old (49 participants), and over 60 of age (7 participants). $81 \%$ of the participants were from one group (20-40). Based on gender, participants were categorized into two groups; male (318 participants/71.8\%) and female (125 participants/28.2\%). Based on education level, participants were categorized as a secondary school (5 participants), high school (28 participants), university graduated (327 participants), and postgraduate (83 participants).

In the sixth question, the survey estimated information about how this population performed their jobs during the break period, and the answers were as following; 126 participants $(24.4 \%$ of the participants) get a break from work during the quarantine period, 173 (39.1\% of the participants) perform their jobs from home, whereas 144 of the participants (32.5\% of the population) were performed partial or full-time jobs. Most of the participants who performed the partial or full-time job were between 20 and 60 of age, no one of over 60 of age in our study performs any outdoor job during the quarantine.

In the second part of the questionnaire, the goal from the questions was to evaluate the level of Arab population knowledge related to the COVID-19 and all the recommendations which are very important to be understood and followed to prevent the spread of infection with COVID-19.

Concerning the WHO's regulations and instructions, 121 of the participants (37.3\%) don't have enough knowledge related to the risk of the airdrops, and how to avoid this infection. 6 participants out of $443(1.4 \%)$ answered the question as they don't have any idea related to it. 6 participants who replied negatively were in groups of age between 20 and 40 years old, this group who was performing a part-time or full-time during the COVID-19 break. The age and educational level considered a significant factor in determining the level of awareness related to the disease. Concerning wearing gloves, 17 participants $(3.8 \%)$ don't know any information about the World 
Health Organization related to gloves wearing. 422 participants (95.3\%) were believed about the importance of the social distance, and they apply it when they are out of their homes.

When we asked the population if they are updated to all the information related to the COVID-19, 302 of the participation (68.2\%) approved that they are updated. 31 of the participants (7\%) answered that they are not up-to-date. 110 participants $(24.8 \%)$ of the population are not sure if they are updated enough.

Most of this population depends on the internet to get updates and news related to the COVID-19 more than television, newspaper, and printed paper. 225 participants (57.6\%) obtained the required information related to the COVID19 from the websites of the states and the World Health Organization. 137 participants (30.9\%) depend mostly on social media (Facebook, Instagram, and Whatsapp). Most of the population (268 participants/ 60.5\%) found the medical source related to the COVID-19 in the Arabic language sources is enough. 134 of the participants (30.2\%) didn't believe that the Arabic language sources are enough. 41 of the participants $(9.3 \%)$ depend only on un-Arabic sources because they believe that it is more trustworthy.

When we ask the population if they agree with the decision taken to suspend the work in dental clinics except for emergency cases, 312 of the population (70.4\%) answered that this is important and they agree with it. 66 of the population (14.9\%) say that they don't have information about it. 65 of the population (14.7\%) did not believe in the importance of this procedure

Most of the participants in this study (375/84.7\%) complied with the instructions of health organizations and declined to visit dental clinics. 41 of them $(9.3 \%)$ visited the clinic for emergency cases, and 21 patients (4.7\%) visited the clinic to complete the dental treatment which started before the break, 35 patients of the population did not comply to the instruction and continue to visit the dental clinics for esthetic and non-emergency procedures.

\section{Discussion}

The center of the COVID-19 changed from China where the number of the positively tested patients has reduced sharply, and now cases increase in the Eastern Mediterranean Region (EMR). According to the WHO EMR, 19 of the 22 countries/ territories have been affected, mostly in the Gulf States, Turkey, and the Islamic Republic of Iran. ${ }^{14}$ The challenges facing some countries in the EMR, including complex emergencies, compounded by fragile health systems, weak disease surveillance, poor response capacity, and a suboptimal level of public health preparedness, the Ministries of Health of 16 of the 22 countries/territories have developed a national action plan against COVID-19 with the support of the WHO Regional Office for the EMR and other United Nations agencies. ${ }^{14}$ The religious mass crowding events which are expected to take place in July 2020 in some countries of the Eastern Mediterranean Region will pose a risk to public health. ${ }^{14}$ As such, this mass gathering brings the possibility of the propagation of respiratory infections ${ }^{15}$ Especially, that many of the countries of the Eastern Mediterranean Region start to release the outbreak and life starts to be normal partially or fully gradually. In these circumstances, the public should be responsible to continue the preventive procedures like the social distance and general hygiene to be able to avoid a second wave of the COVID-19 infection spread. In addition, laboratory capacities are limited, with 20 of the 22 countries in the EMR having a functioning reference laboratory with the ability to detect and confirm MERS-CoV and other high-threat pathogens. When it comes to the detection of a newly emerging pathogen, a lot of training and guidance with the provision of diagnostic kits and laboratory supplies will be required. ${ }^{14}$

This survey provides an insight into the level of awareness, perception, and attitude of the Arab population who are living in different countries on infection control with a special emphasis on COVID-19 at the time of the outbreak in 2020. This study included a random sample of candidates at different groups of age and different educational backgrounds. Most of the participants were from the age group between 20 and 40 of age, the reason related to the ability of this group of age to be active on websites and social media more than other groups of age. This could be considered as a limitation of this study because over 60 of age is the group under risk of complication and fetal deterioration related to COVER-19. And the elder group of age is the group that is not expected to be up to date for the health precautions. But the only way to reach the participants during the outbreak was the internet and social media. The author expected different results if he had the chance to make a real survey to reach the required groups (elderly people).

Most of the participants were from the university and postgraduate groups regarding the level of education because this group of people was more reachable over social media to be included in the study. Most of the participants show a good adaptation for the precautions concerning isolation and quarantine. 299 participants stayed at home during the COVID19 outbreak by taking a break from jobs or performing their jobs from home. $32.5 \%$ of participants who perform partial or full-time jobs were from 20 to 60 groups of age which consider the less risky age group related to the complications and fetal deteriorations compensate with COVID-19 infection. ${ }^{13}$ Besides that, according to the results of this study, this group of age (2060 ) shows a high level of awareness about transmission methods of the virus and the protected methods to avoid infections.

The estimated incubation period of COVID-19 is up to 14 days. ${ }^{13}$ People in this study varied in their knowledge about the incubation period of the disease, but it is essential to know the right incubation period because of its role in determining the safe period to be out of the quarantine especially when the people will come to normal life partially or completely. ${ }^{13}$ The route of the transition of this disease is expressed by the snowball theory, according to research more than two new cases expected with every new case of COVID-19. ${ }^{16}$ The difficulty of preventing the transition of the infection between the individuals is the probability of transition before the onset of symptoms or very early symptoms. ${ }^{15,16}$ For this reason, the present control method which depends on temperature screening is not a trustful method, and there is a critical need for accurate contact tracing starting from the day before the onset of symptoms as well as strict quarantine measures and monitoring before more chains of contagion are established. ${ }^{17} \mathrm{~A}$ recent study showed that many countries in Africa including some countries that are part of the EMR have the variable capacity to respond to outbreaks and high vulnerability. ${ }^{18}$ Besides the protracted conflicts in many countries in the region, lack of infrastructure, limited resources, inadequate prevention control practices, poor preparedness capacity, and inadequate 
laboratory infrastructures and resources in many countries in the EMR are among the main barriers to adequately detect and respond to COVID-19. ${ }^{17}$ Nsour et al in this study highlights the contribution of EMR's FETPs to the preparedness capacities in countries in the EMR to respond to the current COVID19 threat. ${ }^{17}$ The purpose of the Field Epidemiology Training Program (FETP) is to train the population capacity to detect and respond to health threats and establish local experts in the area of epidemiology. ${ }^{19}$ These programs establish a real-time and real place to practice and focus on building knowledge trainers to contribute to their country's health system to detect, notify, report, and respond to events that threaten the national and international health. The programs include many of the SMR's Countries like Egypt, Iraq, Jordan, Morocco, Pakistan, Saudi Arabia, Sudan, Tunisia, and Yemen. ${ }^{17}$ The FETPs in the EMR has a plan for activities to strengthen countries' preparedness against COVID-19. The FETPs participated actively in airport surveillance; implemented temperature screening at ports of entry; developed communication materials and guidelines; and shared information to health professionals and the public, often with a 24-hour dedicated hotline. However, some countries remain ill-equipped, have the poor diagnostic capacity, and are in need of further capacity development in response to public health threats. It is essential that FETPs continue building the capacity to respond to COVID-19 and intensify support for preparedness and response to public health emergencies.

Despite the findings introduced in this study, it is important to stress that this survey had limitations, including the relatively low response rate, which resulted in a smaller than expected sample size. This could have been caused by a short period of data collection and the limitation in the online methods for reaching the participants over the social media platforms. However, this is considered a moderate sample size.

\section{Conclusion}

The majority of the participants had heard about COVID19 and were aware of the infection control measures. Most of the participants strictly adapted to quarantine during the outbreak, and the young group of age who was in needs to perform the job during the outbreak have a good knowledge related to COVID-19 and the method of protection. Further steps need to be taken to enhance the social media accounts and internet websites in the Arabic language which concern medical and educational content. There is currently no standard therapy for COVID-19 and the proposal of digital tools for the contact tracing ready to be applied related to security and privacy issues. So, self-awareness and following health precautions are very important.

\section{Abbreviations}

EMR: Eastern Mediterranean Region FETP: Field Epidemiology Training Program MERS: Middle East Respiratory Syndrome
MERS-CoV: Middle East Respiratory Syndrome Coronavirus SARS: Severe Acute Respiratory Syndrome

WHO: World Health Organization

\section{References}

1. Meng L, Hua F, Bian Z. Coronavirus disease 2019 (COVID-19): Emerging and future challenges for dental and oral medicine. J Dent Res. 2020;99:481487.

2. de Wit E, van Doremalen N, Falzarano D, Munster VJ. SARS and MERS: Recent insights into emerging coronaviruses. Nat Rev Microbiol. 2016;14:523-534.

3. Yin Y, Wunderink RG. MERS, SARS and other coronaviruses as causes of pneumonia. Respirology. 2018;23:130-137.

4. Lu H, Stratton CW, Tang Y. Outbreak of pneumonia of unknown etiology in Wuhan, China: The mystery and the miracle. J Med Virol. 2020. pp. 401-402. doi:10.1002/jmv.25678

5. World Health Organization. Who Global Report on Traditional and Complementary Medicine 2019. World Health Organization; 2019

6. Chen N, Zhou M, Dong X, Qu J, Gong F, Han Y, et al. Epidemiological and clinical characteristics of 99 cases of 2019 novel coronavirus pneumonia in Wuhan, China: A descriptive study. The Lancet. 2020. pp. 507-513. doi:10.1016/s0140-6736(20)30211-7

7. Wang D, Hu B, Hu C, Zhu F, Liu X, Zhang J, et al. Clinical characteristics of 138 hospitalized patients with 2019 novel Coronavirus-infected pneumonia in Wuhan, China. JAMA. 2020. p. 1061. doi:10.1001/jama.2020.1585

8. Sohrabi C, Alsafi Z, O'Neill N, Khan M, Kerwan A, Al-Jabir A, et al. World Health Organization declares global emergency: A review of the 2019 novel coronavirus (COVID-19). Int J Surg. 2020;76:71-76.

9. Research CM, Case medical research. Xiyanping injection for the treatment of new Coronavirus infected pneumonia. Case Medical Res. 2020. doi:10.31525/ct1-nct04275388

10. El-Tholotha M, Bau HH, Song J. A single and two-stage, closed-tube, molecular test for the 2019 novel Coronavirus (COVID-19) at home, clinic, and points of entry. doi:10.26434/chemrxiv.11860137.v1

11. Parry J. China coronavirus: Hong Kong health staff strike to demand borde closure as city records first death. BMJ. 2020. p. m454. doi:10.1136/bmj. m454

12. World Health Organization. WHO Guidelines on Hand Hygiene in Health Care: First Global Patient Safety Challenge: Clean Care is Safer Care. World Health Organization; 2009

13. Abbott S, Hellewell J, Munday J, Funk S, CMMID nCoV working group. The transmissibility of novel Coronavirus in the early stages of the 2019-20 outbreak in Wuhan: Exploring initial point-source exposure sizes and durations using scenario analysis. Wellcome Open Research. 2020. p. 17. doi:10.12688/wellcomeopenres.15718.1

14. Al-Tawfiq JA, Memish ZA. COVID-19 in the Eastern Mediterranean Region and Saudi Arabia: prevention and therapeutic strategies. Int J Antimicrob Agents. 2020;55:105968.

15. Benkouiten S, Al-Tawfiq JA, Memish ZA, Albarrak A, Gautret P. Clinical respiratory infections and pneumonia during the Hajj pilgrimage: $A$ systematic review. Travel Med Infect Dis. 2019;28:15-26.

16. Wu JT, Leung K, Leung GM. Nowcasting and forecasting the potential domestic and international spread of the 2019-nCoV outbreak originating in Wuhan, China: A modelling study. The Lancet. 2020. pp. 689-697. doi:10.1016/s0140-6736(20)30260-9

17. Nsour MA, Al Nsour M, Bashier H, Al Serouri A, Malik E, Khader Y, et al. The role of the global health development/Eastern Mediterranean Public Health Network and the Eastern Mediterranean Field Epidemiology Training Programs in preparedness for COVID-19. JMIR Public Health Surveill. 2020. p. e18503. doi:10.2196/18503

18. Gilbert M, Pullano G, Pinotti F, Valdano E, Poletto C, Boëlle P-Y, et al. Preparedness and vulnerability of African countries against importations of COVID-19: A modelling study. Lancet. 2020;395:871-877.

19. White ME, McDonnell SM, Werker DH, Cardenas VM, Thacker SB. Partnerships in international applied epidemiology training and service, 1975-2001. Am J Epidemiol. 2001;154:993-999. 